\title{
On the Comparison of Various Overhead Arrangements for Massive MIMO-OFDM Channel Estimation
}

\author{
Pallaviram Sure and Chandra Mohan Bhuma
}

\begin{abstract}
Massive multi input multi output (MIMO) systems incorporate orthogonal frequency division multiplexing (OFDM) technology to render high data rate services for future wireless communication applications. The channel estimator (CE) employed by a reliable massive MIMO-OFDM system requires huge amount of overhead in the form of known and null data transmissions, hence limiting the system spectral efficiency (SE). Often, $\mathrm{CE}$ design is a tradeoff between $\mathrm{SE}$ and system reliability. In this paper, $\mathrm{CE}$ with three different overhead arrangements, namely time domain synchronous (TDS), comb type with cyclic prefix (CTCP), $2 D$ grid type with cyclic prefix (GTCP) are investigated and a GTCP based $C E$ is proposed which offers both high SE and improved system reliability. The proposed $\mathrm{CE}$ uses autocorrelation based denoising threshold for channel impulse response (CIR) estimation and does not require any knowledge of channel statistics (KCS). A $4 \times 16$ MIMO-OFDM system is simulated in a rayleigh fading channel environment with U-shaped doppler spectrum. From the bit error rate (BER) performance results in WiMax SUI-4, Advanced Television Technology Center (ATTC) and Brazil A channel environments, it is verified that the proposed CE with GTCP overhead and proposed denoising scheme, indeed improves both SE and system reliability. Hence it is suitable for application in all massive MIMO-OFDM systems.
\end{abstract}

Keywords-Massive MIMO-OFDM, time domain synchronous, comb type with cyclic prefix, grid type with cyclic prefix, denoising threshold, LS channel estimation.

\section{INTRODUCTION}

M ASSIVE MIMO-OFDM technology provides higher data rates for wireless communications. The current wireless standards like 3GPP Long Term Evolution (LTE) and IEEE $802.11 n$ address a small number of transmit antennas (less than tens). But if large number i.e. tens of antennas are used, the spectral efficiency can be improved and higher data rates can be obtained. The current WiFi standards are proceeding towards large MIMO [1]. An investigation on key technologies in large scale MIMO [2] shows that CE is one of the key challenges due to its high dimensionality. For a better estimation and hence system reliability in terms of BER performance, the SE must be sacrificed.

SE is the ratio of useful data bits transmitted to the total data bits transmitted. SE dictates the amount of overhead to be used in CE of any system. The significance of SE is further

Pallaviram Sure is with REVA Institute of Technology and Management, Bangalore, India (e-mail: pallavi@ revainstitution.org).

Chandra Mohan Bhuma is with Bapatla Engineering College, Bapatla (e-mail: chandrabhuma@gmail.com). illustrated in [3]. In a single input single output (SISO)OFDM system, SE is rarely accounted because overhead is in the form of only the known data to be transmitted. On the other hand, in a $Z \times Z$ MIMO-OFDM system, as $Z$ increases, both the known data and the null data to be transmitted increase rapidly and severely effect the SE. So SE is a critical factor in devising CE techniques for massive MIMO-OFDM systems. Various overhead arrangements, available in the literature are discussed below.

In a OFDM system with TDS overhead, data is sent in frames. Each frame has preamble and postfix sequences as overhead. At the receiver, Least Squares (LS) based estimation using the received and the known preamble is performed. A Tail Cancelation and Cyclic Restoration (TCCR) is performed on the received data using the postfix sequences to obtain cyclically reconstructed data [4]. The channel estimates and the reconstructed received data, are used by the detector to compute the estimates of the transmitted data symbols. Some TDS approaches are given in [4], [5]. In [4], a Cazac sequence is used as both preamble for CE. In [5], PN sequences act as preamble, which is used in the $\mathrm{CE}$ and also in cyclic reconstruction of the data. In a $Z \times Z$ MIMO-OFDM system, for all the $Z$ transmitters, $Z$ orthogonal CAZAC sequences are devised. At each of the $Z$ receivers, $Z$ transmit channels are estimated with the help of these orthogonal sequences, and received data is cyclically reconstructed. The total $Z^{2}$ channel estimates and the $Z$ reconstructed received data symbols at a given time instant are given to a high dimensional detector [6], to obtain the estimates of transmitted data symbols.

In a OFDM system with CTCP overhead, a CP inserted at the start of every OFDM symbol forms a part of the overhead. Also, a few or more known symbols called pilots [7], zero transmissions called nulls are spread uniformly across the time frequency grid of OFDM form the rest of overhead. At the receiver, LS based CE is carried out at the pilot positions, using received and pilot symbols. No KCS is required in this estimation. These estimates are interpolated and are given to a detector along with the received symbols. The detector outputs the estimates of transmitted data symbols. In a $Z \times Z$ MIMO-OFDM system, each of the $Z$ transmitters uses a different set of subcarriers for pilot transmissions. Also, nulls are transmitted in the subcarrier sets corresponding to other $Z-1$ transmitters. All the $Z$ receivers provide $Z^{2}$ channel estimates and $Z$ received data symbols to the detector to 
obtain estimates of transmitted data symbols. The CTCP and block type cyclic prefix based systems are studied in [8], [9]. GTCP systems use less overhead than the CTCP systems. Also, as they require a time direction interpolator, their usage is restricted in massive MIMO-OFDM systems.

All the above systems use LS based CE. Other than LS based CE, minimum mean square error (MMSE) estimation can also be performed to estimate the channel CIR. MMSE gives better BER performance and hence system reliability than LS, but it requires KCS parameters which are usually unavailable [7]. So LS based estimation is improved using denoising and truncating operations on the CIR. Various denoising thresholds are obtained by minimizing mean square error [10] and maximizing tap detection probability [11] However, such threshold expressions involve KCS parameters like channel length, noise variance, channel tap power, number of multipaths which are estimated separately. To avoid this estimation, the threshold expression should not have KCS parameters. For this purpose, DFT based channel estimators [12] are improved by iterative estimation procedure based on minimization of generalized akakine information criterion (GAIC) [13], a noise variance based time domain thresholding [14], wavelet based denoising [15], universal threshold based denoising [16] can be used. The performance of LS with threshold based denoising is much close to MMSE, based on the threshold used.

This paper introduces a GTCP based CE, which provides both high SE and improved BER performance. For this purpose, a threshold expression which does not involve any KCS parameters is framed using the LS estimated CIR. The length of the channel is also estimated based on this CIR, for truncation operation. The rest of the paper is organized as follows. The TDS overhead based CE system is described in section II. The CTCP overhead based CE system is described in III. The GTCP overhead and autocorrelation based denoising threshold are developed in section III-C. In section IV, the TDS, CTCP and GTCP based CE simulation details and corresponding results for various channel environments are presented. Finally the paper is concluded in section V.

\section{TDS OVERHEAD ARRAngEMENT BASED System}

Consider a basic large MIMO-OFDM model with $N_{t}$ transmit antennas and $N_{r}$ receive antennas, where $N_{t}, N_{r}$ are in tens or more. At each transmit antenna the raw data is mapped using QPSK and is then divided into $N$ parallel data streams, called $N$ OFDM sub-carriers. Each $N$ symbol parallel data stream is called as an OFDM symbol.

\section{A. System Model}

In TDS based transmitter, an $N$ point IDFT is performed on each OFDM symbol to obtain the time domain OFDM symbols. In $F$ consecutive OFDM symbols, a postfix is inserted after every OFDM symbol. The resulting $F$ parallel data streams are converted to serial, which is called a frame. A preamble is attached at the start of the frame as shown in Fig. 2. Here, $N_{c a z}$ and $N_{p r e}$ are the lengths of postfix and preamble sequences. The frames available at each of the $N_{t}$ transmit antennas are sent through the fading channel environment. A space-time coding is generally incorporated at this stage [17], to achieve spatial multiplexing. At each of the $N_{r}$ receivers, AWGN noise gets added to the received data. The received data is processed by a CE followed by a detector and corresponding detected symbols are de-mapped to binary data.

\section{B. CE Technique}

In the TDS based MIMO-OFDM system, unique sequences like Cazac, Zadoof Chu or pseudo noise (PN) sequences are inserted as preamble [4]. Postfix is the last $N_{c a z}$ part of the Cazac preamble sequence, as shown in Fig. 2. At each receive antenna, based on the autocorrelation properties of all received and transmitted preambles, the $N_{t} N_{r}$ channels are estimated. The received preamble of the $k^{t h}$ frame at the $m^{t h}$ antenna is shown in (1), where, $\times$ represents linear convolution and $c_{n, k}$ are the known preambles.

$$
r_{m, k}=\sum_{i=0}^{N_{t}-1} h_{m n, k} \times c_{n, k}+w_{m, k}
$$

Consider an ideal preamble sequence represented as $c_{n}$, given in (2). Its autocorrelation and cross-correlation properties are given in (3) and (4)respectively, where $I$ is identity matrix of $N_{\text {pre }}$ dimension, $(.)^{H}$ represents the hermitian transpose, $(.)^{*}$ is the complex conjugation, (.)| represents the modulo operation, $\otimes$ is the circular correlation operator.

$$
\begin{aligned}
& c_{n}=\left[\begin{array}{lllll}
c_{n, 0} & c_{n, 1} & c_{n, 2} & \ldots & c_{n, N_{p r e}-1}
\end{array}\right]^{T} \\
& R_{c}(b)=\sum_{i=0}^{N_{\text {pre }}-1} c_{n}^{*}(i) c_{n}\left(\left.(i+b)\right|_{N_{\text {pre }}}\right)=\left\{\begin{array}{cc}
N_{\text {pre }} & b=0 \\
0 & b \neq 0
\end{array}\right. \\
& c_{m}^{H} \otimes c_{n}=0
\end{aligned}
$$

Ideal autocorrelation property ensures the accurate estimation of a given CIR, and ideal cross correlation ensures that the other channel interferences are zero and they do not intervene with the CIR being estimated. The CIR estimation for all the channels is performed using (5). Expanding (5) for each $m, n$ on every OFDM symbol $k$, results in $N_{t}-1$ negligible crosscorrelation terms and one significant autocorrelation term. This results in CIR estimation on the OFDM symbol $k$. A DFT operation on (5), gives the required channel frequency response (CFR).

$\hat{h}_{m n, k}=\frac{1}{N_{\text {pre }}} c_{n, k} \otimes r_{m, k}, 0 \leq n \leq N_{t}-1,0 \leq m \leq N_{r}-1$

Longer the preamble sequence, more ideal are their correlation properties and higher is the estimation accuracy. However, this decreases SE of the TDS system. In general, the channel is assumed to be quasi static over a complete frame and a sufficiently long preamble is chosen in the CIR estimation. Then to retain SE, postfix sequences are chosen of short length and do not aid in accurate estimation [4]. The postfixes are 


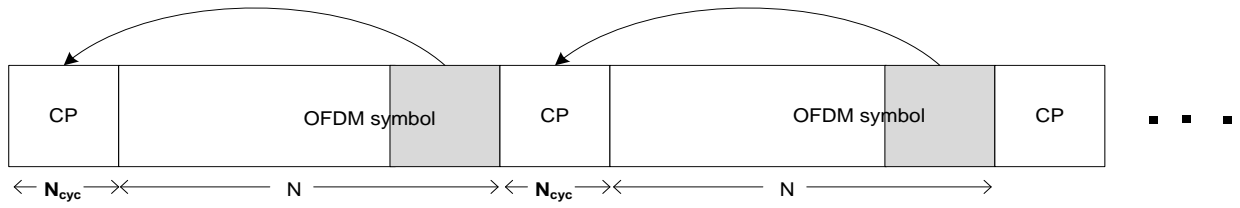

Fig. 1. CP based OFDM system along time direction

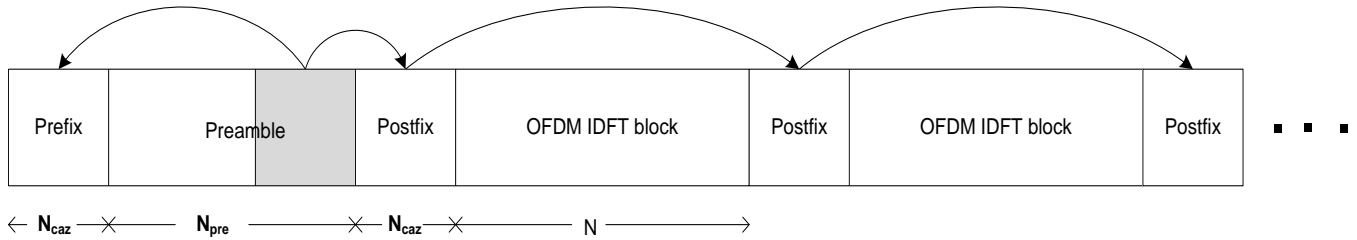

Fig. 2. TDS based OFDM system along time direction

used by the receiver in the process of TCCR. The SE of the TDS based system, is given in (6), for a frame length of $F$ OFDM symbols. $\eta_{0}$ is the SE of an ideal OFDM system with no overhead.

$$
S E=\eta_{0} \frac{F N}{F\left(N+N_{c a z}\right)+N_{p r e}+2 N_{c a z}}
$$

The MIMO detector uses the CIR estimates of all $N_{t} N_{r}$ channels and $N_{r}$ cyclically reconstructed received data symbols, on each subcarrier of every $k^{t h}$ OFDM symbol, to estimate the $N_{t}$ transmitted data symbols. The detectors like zero forcing $(\mathrm{ZF})$, ML or those devised using belief propagation framework can be incorporated. A study on the available detectors is presented in [18].

\section{CTCP OverheAd ARrangement Based System}

Consider a CTCP overhead based system, with $N_{t}$ transmit and $N_{r}$ receive antennas.

\section{A. System Model}

At the transmitter the binary data is mapped to QPSK and is grouped into OFDM symbols, each with $<N$ subcarriers. The remaining subcarriers are reserved for transmitting known data symbols called pilots and also nulls. For a 2 system, the arrangement of data, pilot and nulls is shown in Fig. 3. In Fig. 3 , shaded boxes are pilots, $X$ indicates the null position and $D$ indicates the data position. An $N$ point IDFT is performed on each symbol to obtain the OFDM data symbols in the time domain. Then a CP is inserted at the beginning of each OFDM symbol, which is the last $N_{c y c}$ symbols of the same symbol, as shown in Fig. 1. The CP protects the OFDM symbol from any inter symbol interference (ISI) present in the multipath fading channel. The resultant data is converted to serial and sent through a multipath fading channel. The typical channel impulse response from $p^{\text {th }}$ transmit antenna to $q^{\text {th }}$ receive antenna is modeled as (7) [7]. In (7), $\alpha_{g}^{q, p}$ represents the tap gain of the $g^{t h}$ path and the corresponding path delay is represented as $\tau_{g}^{q, p}$. The tap gain is a complex Gaussian random variable with zero mean and variance $\sigma_{g, q, p}^{2}$.

$$
h^{q, p}(n)=\sum_{g=0}^{L E N-1} \alpha_{g}^{q, p} \delta\left(n-\tau_{g}^{q, p}\right)
$$

At each of the $N_{r}$ receivers, AWGN (Additive White Gaussian Noise) gets added to the received data. The receiver converts the serial data to parallel form and the CP is removed. An $N$ point DFT is performed to obtain frequency domain OFDM symbols. These are sent to CE for channel estimation.

\section{B. CE Technique}

The following notations are used in further representations.

- Received signal at $m^{t h}$ receive antenna, in the $k^{t h}$ OFDM symbol is notated as $Y_{m, k}$, where $Y_{m, k}=$ $\left[\begin{array}{llll}Y_{m, k}(0) & Y_{m, k}(1) & \ldots & Y_{m, k}(N-1)\end{array}\right]^{T}$ represents the received data in frequency domain on $N$ OFDM subcarriers of the $k^{t h}$ OFDM symbol.

- The transmitted symbol from the $n^{\text {th }}$ transmit antenna in $k^{t h}$ OFDM symbol is $X_{n, k}$, where $X_{n, k}=$ $\left[\begin{array}{llll}X_{n, k}(0) & X_{n, k}(1) & \ldots & X_{n, k}(N-1)\end{array}\right]^{T}$, represents the transmitted data in frequency domain on $N$ OFDM subcarriers of the $k^{\text {th }}$ OFDM symbol

- The channel from the $n^{t h}$ transmit antenna to $m^{t h}$ receive antenna is $H_{m n, k}$, where $H_{m n, k}=$ $\left[\begin{array}{llll}H_{m n, k}(0) & H_{m n, k}(1) & \ldots & H_{m n, k}(N-1)\end{array}\right]^{T}$ represented the channel CFR coefficients on $N$ OFDM subcarriers of the $k^{\text {th }}$ OFDM symbol

- Similarly the IDFT of $Y_{m, k}, X_{n, k}$ and $H_{m n, k}$ are represented as $y_{m, k}, x_{n, k}$ and $h_{m n, k}$.

Consider the CTCP arrangement with a transmit sequence $X_{n, k}(a), 0 \leq n \leq N_{t}-1$ for the $k^{t h}$ OFDM symbol of the $n^{\text {th }}$ transmit antenna. The set of sub-carriers $\{a\}$ carrying pilot symbols is not same for all the antennas. For $n^{\text {th }}$ antenna, the subcarrier index $a$ takes the values, $\left\{n, n+S_{f}, n+2 S_{f}, \ldots\right\}$, where $S_{f}$ is the spacing between the pilot sub-carriers. Thus, on a single sub-carrier, if the $n^{t h}$ antenna is transmitting pilots, the rest of the antennas transmit nulls on that sub-carrier, as shown in Fig. 3. At each receive antenna, the received pilot symbols at known positions are used for channel estimation. 
At each receive antenna, in each OFDM symbol, the CFR is estimated at the pilot positions using LS estimation. This CFR is low-pass interpolated along the OFDM symbol and the CFR coefficients at the data and null positions are obtained, to have $N$ length CFR. Some other interpolation techniques are discussed in [7]. Consider the $k^{\text {th }}$ OFDM symbol received at $m^{\text {th }}$ antenna given in (8). In (8), $X_{i, k}^{D}$ is the diagonal matrix formed by $X_{i, k}$. The LS channel estimates before and after interpolation are given in (9), and (10) respectively. Here, $\hat{H}_{m n, k}$ is estimated CFR between $n^{t h}$ transmit and $m^{t h}$ receive antenna on $k^{\text {th }}$ OFDM symbol. Corresponding CIR is $\hat{h}_{m n, k}$.

$$
Y_{m, k}=\sum_{i=1}^{N_{t}} H_{m i, k} X_{i, k}^{D}+W_{m, k}
$$

where, $0 \leq m \leq N_{r}-1$

$$
\begin{gathered}
\hat{H}_{m n, k}(a)=\frac{Y_{m, k}(a)}{X_{n, k}^{D}(a)}, a \in\left\{n, n+S_{f}, n+2 S_{f}, \ldots\right\} \\
\hat{H}_{m n, k}(a)=\frac{Y_{m, k}(a)}{X_{n, k}^{D}(a)}, 0 \leq a \leq N-1
\end{gathered}
$$

Note that for MIMO channel estimation, the pilots are arranged such that when the one of the transmit antenna is sending pilots, the other antennas transmit nulls. So all $X_{i, k}$ are nulls except $X_{n, k}$, which is the pilot sequence. Therefore (8) can be reduced to (11), which is a SISO representation between $m^{t h}$ receive antenna and $n^{t h}$ transmit antenna.

$$
Y_{m, k}=H_{m n, k} X_{n, k}^{D}+W_{m, k}
$$

The time domain equation corresponding to (10) by substituting (11) in (10), is given in (12), where $v_{m, k}(a)$ is the modulated noise term. The second term of (12), shows that the channel estimates of (12) are noisy.

$$
\hat{h}_{m n, k}(a)=h_{m n, k}(a)+v_{m, k}(a), 0 \leq a \leq N-1
$$

The CIR of the channel is $\hat{h}_{m n, k}$ of (12) and corresponding CFR is $H_{m n, k}$. The amount of pilot sub-carriers required in CTCP overhead is almost same as the number of data subcarriers [1], which is very large overhead. Consider a $4 \times 4$ system in which, each antenna 1 transmits pilots on sub-carrier 1. Then antenna 1 should always send nulls on 2,3 and 4 sub-carriers. So it is a huge wastage, since data is not being transmitted. Similar is the case with all the 4 antennas in this system. The SE of CTCP based system is given in (13). Here, $G$ is the number of OFDM symbols.

$$
S E=\eta_{0} \frac{\left(S_{f}-N_{t}\right) G}{\left(S_{f}-N_{t}\right) G+N_{t} G}=\eta_{0} \frac{\left(S_{f}-N_{t}\right)}{S_{f}}
$$

The detector is same as that for TDS case, except that it uses the CFR estimates of all $N_{t} N_{r}$ channels and $N_{r}$ received data symbols. No cyclic reconstruction is necessary on the received symbols due to $\mathrm{CP}$ protection. Comparing TDS based system with CTCP based system, it is clearly understood that a TDS system has better SE than a CTCP system. Also performance of TDS based system relies on both CE and TCCR unlike that of CTCP system which relies on CE alone. The number of pilots in a CTCP system is much higher and more frequent

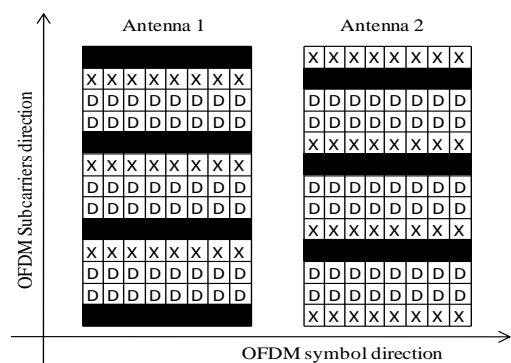

Fig. 3. CTCP overhead arrangement for $2 \times 2$ MIMO-OFDM system

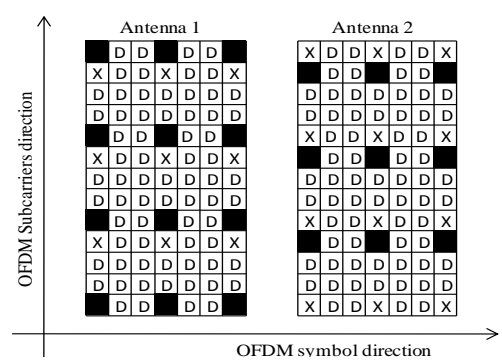

Fig. 4. GTCP overhead arrangement for $2 \times 2$ MIMO-OFDM system

than that in TDS system. So CTCP system performance is more reliable than that of a TDS based system.

\section{GTCP Overhead Arrangement Based System}

Usually, the wireless channel is assumed to be of slow fading type, when it comes to MIMO-OFDM systems. But in general the channel may be of any type [1]. If the channel is of fast fading type, the comb type is the most preferred pilot arrangement. But as seen from previous paragraph, the amount of overhead required is very large in such systems. In massive MIMO-OFDM systems, as overhead or SE is of major concern, a GTCP is a suitable choice. The GTCP uses same system model as a CTCP except for the pilot arrangement. In this system, at each transmit antenna, pilots are sent in the OFDM symbols from the set $\left\{k, k+G_{t}, k+2 G_{t}, \ldots\right\}$, where $G_{t}$ is the spacing between adjacent pilot symbols on a single subcarrier. The $n^{t h}$ transmit antenna sends pilots on the sub-carriers $\left\{n, n+S_{f}, n+2 S_{f}, \ldots\right\}$, the $(n+1)^{t h}$ antenna sends pilots on $\left\{n+1, n+1+S_{f}, n+1+2 S_{f}, \ldots\right\}$ where $S_{f}$ is the spacing between the pilot sub-carriers in $k^{t h}$ OFDM symbol. This arrangement is shown for a $2 \times 2$ system in Fig. 4. As in CTCP system, when one antenna transmits pilots, the others transmit nulls in those corresponding positions. The pilot pattern at a given transmit antenna resembles a $2 D$ grid type pattern. In this system, after LS channel estimation at pilot positions, interpolation is required at each receive antenna in both time and frequency directions, i.e. along the symbol and along the subcarrier directions. For frequency interpolation, a low pass interpolator is used and for time interpolation, a simple linear interpolator is used to keep the computational complexity at a minimal level. After LS estimation and both the interpolations, the channel estimates are given by (10). Correspondingly, because a single antenna transmits pilots, while others transmit nulls, the CIR after 
time and frequency direction interpolations becomes (12). However as GTCP arrangement has higher SE than a CTCP, the BER performance may degrade. To improve on this aspect, a denoising and truncation are performed on (12). Denoising the channel estimates, with a threshold is given in (14). Truncating the CIR with the channel length as $L E N$, the final channel estimates are given in (15). The threshold $\gamma$ used in (14) is described later.

$$
\begin{gathered}
\hat{h}_{m n, k}(a)=\left\{\begin{array}{cc}
\hat{h}_{m n, k}(a), & \left|\hat{h}_{m n, k}(a)\right|^{2} \geq \gamma \\
0, & \text { otherwise }
\end{array}\right. \\
\hat{h}_{m n, k}(a)=\left\{\begin{array}{cc}
\hat{h}_{m n, k}(a), & 0 \leq a \leq L E N-1 \\
0, & L E N \leq a \leq N-1
\end{array}\right.
\end{gathered}
$$

The SE of this system, is given in (16), where $G$ represents the spacing between two adjacent pilot OFDM blocks. $N$ is the number of sub-carriers, $N_{c y c}$ is the length of the cyclic prefix.

$$
S E=\eta_{0} \frac{\left(G_{t}-1\right) N+\left(S_{f}-N_{t}\right) \frac{N}{S_{f}}}{G_{t}\left(N+N_{c y c}\right)}
$$

Consider the SE of this system by choosing $G_{t}=F+1$, $N_{c y c}=N_{c a z}, N=N_{\text {pre }}+N_{c a z}$. Then SE reduces to that of TDS system except for the extra term $\left(S_{f}-N_{t}\right) \frac{N}{S_{f}}$ in the numerator. This contributes to increased SE in a TDS system. However, if $S_{f}=N_{t}$, then this term becomes zero and the SE of GTCP is same as that of a TDS system. The SE of GTCP system varies with $N_{t}$ unlike that of a CTCP or a TDS system due to the term $\left(S_{f}-N_{t}\right) \frac{N}{S_{f}}$ in the numerator. The detector in a GTCP is same as a CTCP detector.

\section{Proposed Denoising Threshold}

Assuming the KCS are completely unknown, let the estimated CIR, $\hat{h}_{m n, k}$ with $N$ length be notated as $\hat{h}(a), 0 \leq$ $a \leq N-1$. The estimate of autocorrelation function of $\hat{h}$ is computed as in (17) for $M$ delays, where $M \approx \frac{N}{5}$.

$$
\hat{r}_{\hat{h}}(p)=\frac{1}{N} \sum_{a=p+1}^{N} \hat{h}(a) \hat{h}(a-p), 0 \leq p \leq M-1
$$

The denoising threshold is chosen as $\gamma=\hat{r}_{\hat{h}}(0)$ as it indicates the average power of the LS estimated and interpolated channel CIR. The autocorrelation function decays as a function of $p$. The value of $p$ at which this correlation decays to almost a constant minimum value, is the estimated channel length $L E N$ used in truncation of CIR, as in (15). Note that instead of the proposed threshold, any other threshold can be used, however it should have low computational complexity and should suit for unavailable KCS conditions for application in massive MIMO-OFDM systems. The proposed threshold uses only $L E N$ estimation and involves no other KCS parameters. It is framed as a compromise for complexity and good BER performance. If the complexity needs to be further decreased, the length can be chosen arbitrarily high, which introduces a little degradation in BER performance.

\section{Simulation Results AND Discussion}

From the system models discussed above, it is understood that a GTCP is better than a CTCP system in terms of SE. However, the SE of GTCP and TDS are not compared. In the following system simulations, the preamble and the postfix lengths of TDS, total pilots and CP length in GTCP for $F$ OFDM symbols are appropriately chosen such that $G_{t}=F+1, N_{p r e}+N_{c a z}=N$ and $N_{c y c}=N_{c a z}$. With this choice on system overhead, a $4 \times 16$ MIMO-OFDM configuration is simulated. The mapper is QPSK, Space Time Block coding (STBC) adopted is Alamouti STBC scheme [17]. The elementary symbol period in an OFDM symbol is $0.109375 \mu \mathrm{s}$. The Maximum Likelihood (ML) detector is incorporated for detection. The other details of the system parameters used in simulations are shown in Table I. The frame length used in a TDS system is 4 OFDM symbols. The fraction of bits used for carrying overhead information is given in the table, which is notated as $\frac{S E}{\eta_{0}}$. The table shows that the SE of GTCP is $12 \%$ more than a TDS system. If $N_{t}=16$, keeping all the other parameters same, the SE of a GTCP is same as that of TDS system.

There are a total 64 channels in the simulated MIMO configuration. They are identically distributed rayleigh fading channels, each of them is characterized by correlation between CIR samples. So each fading channel is simulated to have a U-shaped power spectral density according to the procedure in [19]. The maximum doppler frequency is $20 \mathrm{~Hz}$ in these simulations. The power delay profiles chosen are those of WiMax SUI4, ATTC (Advanced Television Technology Center) [7], and Brazil-A channels.

The BER performance plots of the three overhead arrangements TDS, CTCP and GTCP with proposed denoising threshold are obtained in each of the channel environments. In ATTC, WiMax SUI4, Brazil-A channel environments, the BER plots are shown in Fig. 5, Fig. 6, Fig. 7 respectively. The BER performance is a plot of BER as a function of input signal to noise ratio (SNR) at the receiver. The performance plots for a TDS, CTCP and the GTCP with proposed denoising strategy are simulated for each channel environment. The benchmark in these plots is the BER performance plot when the receiver has perfect knowledge of the channel. The results show that the GTCP system performs much better than the CTCP and TDS systems in terms of BER. Also for a given amount of data, the GTCP has $12 \%$ better SE than TDS and $26 \%$ better SE than the CTCP system as given in Table. I.

The performance degradation of TDS based system is attributed to the TCCR step required along with channel estimation before detection. The performance of CTCP system is better due to the $\mathrm{CP}$, which removes the necessity of TCCR. However, its SE is poor compared to TDS system. The GTCP system with the proposed denoising scheme combines the advantages of TDS and CTCP. It has better BER performance than CTCP system and better SE than TDS system. The computational complexity of the GTCP system with proposed denoising increases the computational complexity only slightly 
TABLE I

SYSTEM PARAMETERS USED IN SIMULATION

\begin{tabular}{|c|c|c|c|}
\hline Parameters / Overhead arrangement & CTCP & TDS & GTCP \\
\hline Number of Subcarriers, $N$ & 1024 & 1024 & 1024 \\
\hline Pilot spacing in frequency direction, $S_{f}$ & 32 & - & 16 \\
\hline Pilot spacing in time direction, $G_{t}$ & - & 5 & - \\
\hline Number of OFDM symbols in a frame, $F$ & - & - & 4 \\
\hline Type of the Gaurd interval & Cyclic Prefix & Postfix & Cyclic Prefix \\
\hline Length of the Prefix/Postfix & 256 & 256 & 256 \\
\hline Preamble Length $N_{p r e}$ & - & 768 & - \\
\hline$\frac{S E}{\eta_{0}}$ & 0.5 & 0.64 & 0.76 \\
\hline Channel Type & \multicolumn{3}{|c|}{ ATTC, WiMax SUI4, Brazil-A $20 \mathrm{~Hz}$} \\
\hline Channel environment & \multicolumn{4}{|c}{} \\
\hline
\end{tabular}

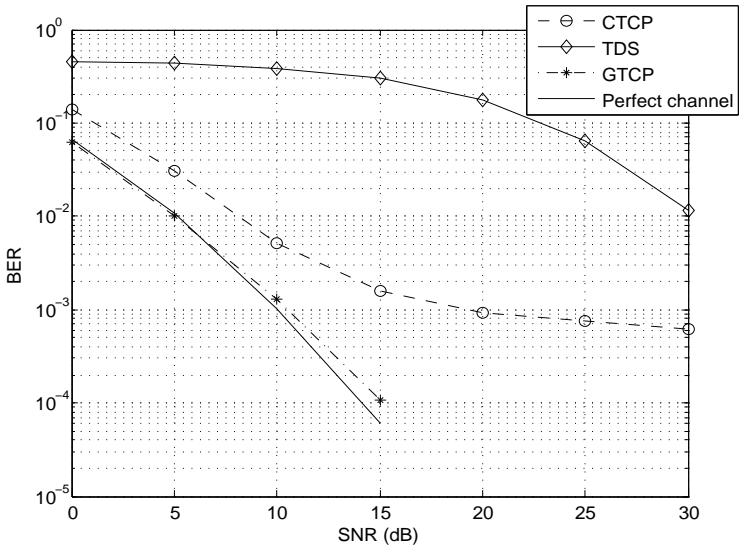

Fig. 5. BER performance of $16 \times 16$ MIMO system in ATTC channel environment

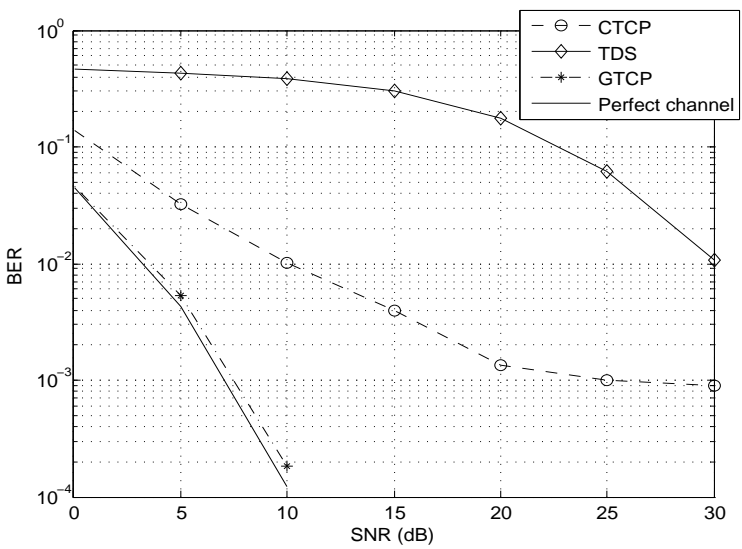

Fig. 6. BER performance of $16 \times 16$ MIMO system in WiMax SUI4 channel environment

due to the time interpolator. Thus it is a suitable system for all practical massive MIMO-OFDM systems.

\section{CONCLUSION}

For massive MIMO-OFDM systems three overhead arrangements, TDS, CTCP and GTCP are investigated. The TDS based system has higher SE, but poor BER performance. The CTCP based system has better BER performance than a TDS system, but SE degrades. Therefore, a GTCP system which maintains the high SE and offers improved BER performance than TDS and CTCP systems is introduced with a proposed

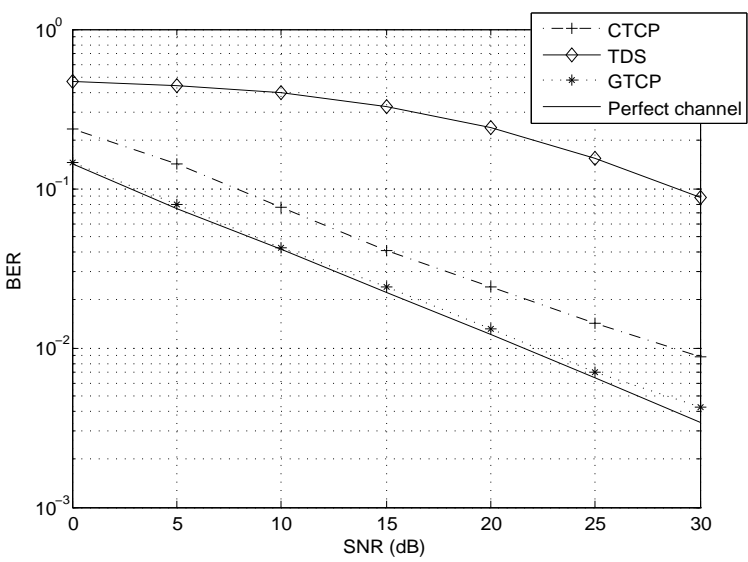

Fig. 7. BER performance of $16 \times 16$ MIMO system in Brazil-A channel environment

denoising threshold. A $4 \times 16$ MIMO-OFDM configuration is simulated with identical channels of WiMax SUI4, ATTC and Brazil-A type environments. In all the three environments, the three overhead arrangements are used. The BER performance of the GTCP is verified to be indeed better than the TDS and CTCP systems and its SE is also higher than the other two systems. This investigation shows that the GTCP based CE with proposed denoising is a suitable technique for reliable massive MIMO-OFDM systems.

\section{REFERENCES}

[1] L. Dai, Z. Wang, and Z. Yang, "Spectrally efficient time-frequency training ofdm for mobile large-scale mimo systems," Selected Areas in Communications, IEEE Journal on, vol. 31, no. 2, pp. 251-263, 2013.

[2] X. Su, J. Zeng, L.-P. Rong, and Y.-J. Kuang, "Investigation on key technologies in large-scale mimo," Journal of Computer Science and Technology, vol. 28, no. 3, pp. 412-419, 2013. [Online]. Available: http://dx.doi.org/10.1007/s11390-013-1342-4

[3] H. Q. Ngo, E. G. Larsson, and T. L. Marzetta, "Energy and spectral efficiency of very large multiuser mimo systems," CoRR, vol. abs/1112.3810, 2011.

[4] L. Dai, Z. Wang, and S. Chen, "A novel uplink multiple access scheme based on tds-fdma," Wireless Communications, IEEE Transactions on, vol. 10, no. 3, pp. 757-761, 2011.

[5] J. W. Z. T. P. M. M. Dai, L Wang, "Time domain synchronous ofdm based on simultaneous multi-channel reconstruction," ser. Proc. of the IEEE International Confence on Communications, 2013, pp. 1-5.

[6] S. K. Mohammed, A. Zaki, A. Chockalingam, and B. S. Rajan, "Highrate space-time coded large mimo: Low-complexity detection and channel estimation," CoRR, vol. abs/0809.2446, 2008.

[7] S. Coleri, M. Ergen, A. Puri, and A. Bahai, "Channel estimation techniques based on pilot arrangement in ofdm systems," Broadcasting, IEEE Transactions on, vol. 48, no. 3, pp. 223-229, 2002. 
[8] K. Zheng, J. Su, and W. Wang, "Iterative dft-based channel estimation for mimo-ofdm systems," in Communications, Circuits and Systems Proceedings, 2006 International Conference on, vol. 2, 2006, pp. 10811085.

[9] W. Li, X. Wang, P. Gu, and D. Wang, "Research on channel estimation of mimoofdm system," in Informatics and Management Science III, ser. Lecture Notes in Electrical Engineering, W. Du, Ed. Springer London, 2013, vol. 206, pp. 67-73.

[10] S. Rosati, G. Corazza, and A. Vanelli-Coralli, "Ofdm channel estimation based on impulse response decimation: Analysis and novel algorithms," Communications, IEEE Transactions on, vol. 60, no. 7, pp. 1996-2008, 2012.

[11] J. Oliver, R. Aravind, and K. M. M. Prabhu, "Sparse channel estimation in ofdm systems by threshold-based pruning," IEEE Electronics Letters, vol. 44, no. 13, pp. 830-832, 2008.

[12] S. M. v. d. B. J.-J. W. S. B. Edfors, O., "Analysis of dft based channel estimators for ofdm," Wireless Personal Communications, vol. 12, pp. $55-70,2000$

[13] M. R. Raghavendra and K. Giridhar, "Improving channel estimation in ofdm systems for sparse multipath channels," IEEE Signal Processing Letters, vol. 12, no. 1, pp. 52-55, 2005.
[14] Y. Kang, K. Kim, and H. Park, "Efficient dft-based channel estimation for ofdm systems on multipath channels," Communications, IET, vol. 1, no. 2, pp. 197-202, 2007.

[15] Y.-S. Lee, H.-C. Shin, and H. N. Kim, "Channel estimation based on a time-domain threshold for ofdm systems," Broadcasting, IEEE Transactions on, vol. 55, no. 3, pp. 656-662, 2009.

[16] H. Xie, G. Andrieux, Y. Wang, J.-F. Diouris, and S. Feng, "Efficient time domain threshold for sparse channel estimation in \{OFDM\} system," AEU- International Journal of Electronics and Communications, pp. -, 2013.

[17] I. Choi, J.-K. Kim, H. Lee, and I. Lee, "Alamouti-codes based fourantenna transmission schemes with phase feedback," Communications Letters, IEEE, vol. 13, no. 10, pp. 749-751, October 2009.

[18] W. Y. Y.-C. G. K. Yong Soo Cho, Jaekwon Kim, MIMO OFDM Wireless Communications with MATLAB. Wiley, 2010

[19] L. Zhinian and Z. Wenjun, "Simulation for correlated rayleigh fading channels by fir pulse-shaping filtering," in Wireless Communications, Networking and Mobile Computing, 2007. WiCom 2007. International Conference on, 2007, pp. 1091-1094. 